\title{
Mortalidade Materna em Recife. Causas de Óbitos Maternos
}

\author{
Maternal Mortality in Recife. \\ Causes of Maternal Deaths
}

José Guilherme Cecatti, Rivaldo Mendes de Albuquerque,

Ellen Hardy, Aníbal Faúndes

\begin{abstract}
RESUMO
Este estudo avaliou os casos de óbitos de mulheres com idade de 10 a 49 anos, ocorridos em Recife, Pernambuco, nos anos 1992 e 1993, com a finalidade de identificar as causas de óbitos maternos. As informações foram obtidas a partir de 1.013 declarações de óbito, sendo complementadas com consultas aos prontuários médicos, fichas de anestesia, relatórios de enfermagem, necrópsias e por meio de entrevistas com os médicos que assistiram estes óbitos ou com familiares das mulheres. As principais causas básicas de óbito materno identificadas foram hipertensão arterial (23,8\%), infeccões (19,0\%), aborto (11,9\%), hemorragias (9,5\%), embolia pulmonar (4,8\%) e acidentes anestésicos (2,4\%). Cerca de $70 \%$ das mortes maternas ocorridas em Recife neste periodo foram por causas obstétricas diretas.
\end{abstract}

PALAVRAS-CHAVE: Mortalidade materna. Hipertensão. Infecção puerperal. Aborto. Complicações da gestação.

\section{Introdução}

Entende-se como morte materna aquela que se encontra relacionada direta ou indiretamente com o processo reprodutivo, desde que iniciada a gestação. Inclui também, desta forma, as mortes resultantes de aborto ou prenhez ectópica, além das advindas de complicações do ciclo gravídico-puerperal. Conforme definição da Organização Mundial de Saúde em 1975, e mantida até o presente, morte materna é "a morte da mulher durante a gestação ou no período de até 42 dias após seu término, independente da duração ou localização da gravidez, por qualquer causa

Departamento de Tocoginecologia, Faculdade de Ciências Médicas, Universidade Estadual de Campinas

Fundação Universidade de Pernambuco

Centro de Pesquisas das Doenças Materno-Infantis de Campinas

Correspondência:

José Guilherme Cecatti

Caixa Postal 6030

13083-970 - Campinas - SP relacionada ou agravada pela gestação, ou por medidas tomadas com relação à gestação, excetuando-se porém as causas acidentais ou incidentais" ${ }^{15}$. Mais recentemente, a própria Organização Mundial da Saúde passou a considerar um período mais longo, de até um ano após o término da gestação, para o conceito de morte materna $^{14}$.

A Federação Internacional de Ginecologia e Obstetrícia (FIGO) tem questionado essa definição como não sendo a mais adequada e discutido a importância de se considerar as mortes maternas não-obstétricas, também chamadas de não-relacionadas. Pode-se citar, por exemplo, a morte de uma gestante conseqüente a acidente de trânsito ou após cirurgia de um tumor cerebral. Chega mesmo a recomendar que todas as mortes, independentemente da causa, que ocorram até 42 dias após o término do processo gestacional e todas as mortes nas quais tenha sido possivel identificar como desencadeante o processo gestacional, até um ano pós-parto, devam ser consideradas como óbito materno ${ }^{8,16}$.

A morte materna pode ser classificada em 
dois grupos, o dos óbitos por causas diretas e o por causas indiretas ${ }^{15}$. As mortes obstétricas diretas são aquelas que resultam de complicações obstétricas na gravidez, parto e puerpério, devidas a intervenções, omissões, tratamento incorreto ou de uma seqüência de eventos resultantes de qualquer uma destas situações. As mortes obstétricas indiretas são as que resultam de doenças existentes antes da gravidez ou que se desenvolvem durante a gravidez e que não foram devidas a causas obstétricas diretas, mas que foram agravadas pelos efeitos fisiológicos da gravidez.

A melhoria da assistência à gravidez, parto e puerpério, observada principalmente nos paises desenvolvidos, propiciou uma queda significativa da mortalidade materna provocada por causas obstétricas diretas e uma conseqüente ascensão proporcional das indiretas, determinada pelas doenças intercorrentes, de bem mais difícil controle. Nos Estados Unidos, por exemplo, as causas obstétricas indiretas predominan sobre as diretas como determinantes de óbito marterno ${ }^{9}$.

As causas obstétricas diretas são responsáveis pela maior parte dos óbitos em países em desenvolvimento, apesar da redução ocorrida nas últimas décadas, devida ao aperfeiçoamento da assistência pré-natal, às transfusões sangüíneas, à antissepsia, à utilização dos antibióticos, à anestesia especializada e à melhor condução do parto e puerpério. Entre as causas obstétricas diretas mais incidentes estão as hemorragias, as infecções, as complicações das doenças hipertensivas e o aborto, além das complicações tromboembólicas e acidentes anestésicos ${ }^{5,11}$

Uma das grandes dificuldades para identificação das mortes maternas se deve a qualidade das informações das declarações de óbito, já que este é o instrumento que permite identificar as causas dos mesmos. Em sua maioria, elas são inadequadamente preenchidas, quer pela pouca importância que se dá a este registro, quer por desconhecimento de como preenchê-la, ou mesmo para mascarar a verdadeira causa do óbito. De uma forma geral, o médico, responsável pelo preenchimento da declaração, informa a causa terminal e não a causa básica que levou à morte, omitindo também se a mulher esteve ou estava grávida. Com isto, fica praticamente impossivel identificar a morte como materna. A pesquisa das mortes maternas vê-se também dificultada pela deficiência dos registros hospitalares ${ }^{2,7}$.

O conhecimento atual sobre o número de mulheres que morrem por complicações da gestação, parto e puerpério no Brasil é obtido, na grande maioria das vezes, por estatísticas oficiais, ou por estudos em instituições hospitalares, que não refletem a real magnitude do problema, quer por má qualidade dos registros oficiais, quer por representar situações que não se aplicam à população geral, como é o caso dos hospitais de referência dos grandes centros urbanos. É unânime, em todo o país, a constatação das dificuldades de obtenção de dados oficiais confiáveis sobre a mortalidade materna ${ }^{10}$.

Nos países em desenvolvimento, incluindo Brasil, a quase totalidade dos estudos sobre mortalidade materna é descritiva, com amostras populacionais não necessariamente representativas $^{13}$. No Brasil, dentre os estudos descritivos, pode-se considerar como de maior importância aqueles de base populacional, pois apresentam dados que podem ser melhor generalizados. Entretanto, são escassas as publicações sobre o assunto que utilizam este método ${ }^{3,4,10}$. Um destes estudos, do tipo caso-controle, identificou um maior risco de morte por causa materna em mulheres com patologias associadas à gestação detectadas durante o pré-natal e naquelas cujo parto foi por cesárea ${ }^{4}$

Embora apareça, ao longo do tempo, uma tendência à diminuição da mortalidade materna, ela ainda é muito elevada quando comparada aos países desenvolvidos. Em nosso meio, a maioria dos óbitos maternos decorre de causas diretas, portanto, passiveis de serem evitadas em quase todas as situações ${ }^{6}$.

A avaliação das causas que determinaram mortes maternas, bem como o conhecimento da história destas mortes, deverão contribuir para que as autoridades sanitárias, a sociedade e, em especial, as mulheres, exijam prioridade no atendimento integral à sua saúde, reconhecendo definitivamente esta prática como a mais efetiva para a promoção da saúde reprodutiva e o seu direito à cidadania.

A necessidade de se conhecer a dimensão do problema da morte materna numa região pobre, como é o Nordeste brasileiro, e dentro de uma cidade onde se dispõem apenas de dados de instituições hospitalares, fundamentou a realização deste estudo descritivo de base populacional, que foi o primeiro do nordeste a utilizar esta abordagem.

\section{Material e métodos}

Foram estudados os óbitos de mulheres de 10 a 49 anos de idade, residentes em Recife e que ocorreram neste município, no período de janeiro de 1992 a dezembro de 1993. Os procedimentos e método do estudo já foram detalhadamente descritos em outra publicação ${ }^{1}$.

Os dados foram obtidos a partir das 
declarações de óbito (DO), dos prontuários médicos, das fichas de anestesia, dos relatórios de enfermagem, das perícias tanatoscópicas do Instituto de Medicina Legal (IML), dos relatórios de necrópsias do Serviço de Verificação de Óbitos (SVO) e das entrevistas com médicos ou familiares das mulheres que faleceram.

Nos óbitos em domicílio, inicialmente procurou-se conversar com o médico que preencheu a DO, e quando não se obtiveram mais informações que elucidassem o caso, procedeu-se à visita domiciliar na residência da falecida, esclarecendo-se que as informações obtidas eram confidenciais e seriam utilizadas exclusivamente para este estudo. Após obtidas as informações, procedeu-se à classificação dos óbitos maternos em diretos e indiretos e selecionaram-se suas respectivas causas básicas.

\section{Resultados}

Identificaram-se 1.013 óbitos de mulheres com idade de 10 a 49 anos, residentes no município de Recife e falecidas nesta cidade no período do estudo. Dentre estes, identificaram-se 42 mortes maternas, sendo que vinte e duas não foram registradas como tal nas declarações de óbito, mas identificadas após processo de investigação (Tabela 1). Pode-se observar na Tabela 2 que 71,4\% das 42 mortes maternas foram por causas diretas. Além destas mortes obstétricas, houve onze óbitos por causas acidentais ou incidentais.

Tabela 1 - Identificação de mortes maternas entre as de mulheres em idade fértil. Recife, 1992-1993.

\begin{tabular}{lrrr}
\hline Mortes em idade fértil & 1992 & 1993 & Total \\
\hline Total & 552 & 491 & 1.013 \\
Maternas declaradas & 7 & 13 & 20 \\
Maternas confirmadas & 20 & 22 & 42 \\
\hline
\end{tabular}

Tabela 2 - Distribuição dos óbitos maternos comforme classificação geral das causas. Recife, 1992 - 1993

\begin{tabular}{lcc}
\hline Classificação das causas & $\mathrm{n}^{\circ}$ & $\%$ \\
\hline Maternas obstétricas & 42 & 79,2 \\
Diretas & 30 & 71,4 \\
Indiretas & 12 & 28,6 \\
Maternas não-obstétricas & 11 & 20,7 \\
Total & 53 & 100,0 \\
\hline
\end{tabular}

Em relação às mortes maternas obstétricas, as principais responsáveis foram a hipertensão arterial, com quase um quarto das mortes, e as infecções puerperais, com quase $20 \%$. As complicações de aborto e a hemorragia representaram quase 12 e $10 \%$ das mortes maternas, respectivamente. Todas as mortes por aborto foram decorrentes de interrupção voluntária da gestação (Tabela 3). Houve 11 casos de mortes maternas não-obstétricas, sendo que sete foram mortes violentas e quatro por neoplasia maligna associada ou decorrente da gestação (Tabela 4). Destacam-se entre estas mortes classificadas como não-relacionadas, dois casos de coriocarcinoma e dois suicídios no oitavo mês de gestação.

Tabela 3 - Distribuição das causas básicas de morte materna obstétrica. Recife, 1992 - 1993

\begin{tabular}{lrr} 
Causas básicas & $\mathrm{N}^{\circ}$ & $\%$ \\
\hline Hipertensão & 10 & 23,8 \\
Infecção & 8 & 19,0 \\
Aborto & 5 & 11,9 \\
Hemorragia & 4 & 9,5 \\
Embolia & 2 & 4,8 \\
Acidente anestésico & 1 & 2,4 \\
Causas indiretas & 12 & 28,6 \\
& & 100,0 \\
Total & 42 &
\end{tabular}

Tabela 4 - Distribuição das mortes maternas não-obstétricas por idade gestacional e causa básica. Recife, 1992 - 1993.

Causa básica

Idade gestacional (mês) / Número

Acidente de trânsito (03)

$6^{\circ} /(2)$

Eletroplessão

$5^{\circ} \quad(1)$

Doença de Hodgkin

$7^{\circ} \quad(1)$

Coriocarcinoma (02)

$2^{\circ} \quad(1)$

Suicídio (02)*

$-/(2)$

Homicídio**

$8^{\circ} /(2)$

$4^{\circ} \quad(1)$

Câncer de mama

$7^{\circ} \quad(1)$

* Familiares não localizados

** Morta em discussão após acidente de trânsito 
Guanto à associação de condições patológicas com a gestação, pode-se observar que mais de um terço das mulheres não tinha doenças prévias ao evento que determinou a morte e em $17 \%$ dos casos de morte materna não havia informação sobre esta condição. Ocorreram seis casos de infecção durante a gestação, sendo a metade dos casos por problemas no aparelho respiratório. A hipertensão arterial foi patologia associada em cinco casos de morte materna obstétrica direta. O conjunto das pneumonias, valvulopatias e lúpus foi responsável por metade dos casos de morte materna indireta (Tabela 5).

Tabela 5 - Distribuição das mulheres com patologias associadas nos casos de morte materna, de acordo com a causa básica da morte. Recife, 1992 - 1993.

\begin{tabular}{|c|c|c|c|c|c|c|c|c|}
\hline \multirow[b]{2}{*}{$\begin{array}{l}\text { Patologia } \\
\text { associada }\end{array}$} & \multicolumn{8}{|c|}{ Causa básica de morte } \\
\hline & Hipert. & Infecção & Aborto & Hemor. & Embolia & Ac. Anest. & $\begin{array}{l}\text { Causas } \\
\text { Indiret. }\end{array}$ & Total \\
\hline $\begin{array}{l}\text { Ausência } \\
\text { de patologia }\end{array}$ & 2 & 5 & 5 & 3 & - & 1 & - & 16 \\
\hline Hipertensão & 4 & - & - & 1 & - & - & - & 5 \\
\hline Pneumonia & - & - & - & - & 1 & - & 2 & 3 \\
\hline Lúpus & - & - & - & - & - & - & 2 & 2 \\
\hline Valvulopatias & - & - & - & - & - & - & 2 & 2 \\
\hline $\begin{array}{l}\text { Amnior. prematur } \\
\text { Corioamnionite }\end{array}$ & & 1 & - & - & - & - & - & 1 \\
\hline $\begin{array}{l}\text { Infecção } \\
\text { urinária }\end{array}$ & - & 1 & - & - & - & - & - & 1 \\
\hline $\begin{array}{l}\text { Espancamento/ } \\
\text { Feto morto }\end{array}$ & - & - & - & - & 1 & - & - & 1 \\
\hline Psicose & - & - & - & - & - & - & 1 & 1 \\
\hline Epilepsia & - & - & - & - & - & - & 1 & 1 \\
\hline Neurofibromatose & e 1 & - & - & - & - & - & - & 1 \\
\hline Colecistite & - & - & - & - & - & - & 1 & 1 \\
\hline Ignorada & 3 & 1 & - & - & - & - & 3 & 7 \\
\hline
\end{tabular}

\section{Discussão}

Os resultados deste estudo mostram, como era de se esperar, que as cifras oficiais de mortalidade materna estão subenumeradas, confirmando a necessidade da utilização de fatores de correção para se conhecer a real magnitude do problema em nosso país ${ }^{12}$.

Aproximadamente três quartos das mortes maternas obstétricas foram por causas diretas, entendidas como as decorrentes de complicações obstétricas da gravidez, parto e puerpério. Esta proporção de mortes por causa direta $(80,6 \%)$ foi muito semelhante à observada em Campinas para os anos de 1985-1991 ${ }^{4}$. Por outro lado, foi muito maior que a encontrada na maior parte dos países desenvolvidos, onde há um predomínio de causas indiretas ${ }^{6}$.

A principal causa básica de morte materna obstétrica encontrada neste estudo foi a hipertensão arterial, quer específica da gestação ou crônica. Estes resultados coincidem com a maior parte dos estudos brasileiros ou em outros países em desenvolvimento ${ }^{3,4,12,13}$. Entende-se que uma alta proporção de mortes maternas por hipertensão deva ser atribuida a falhas principalmente na assistência pré-natal, apesar de existir um componente também atribuível à atenção hospitalar ao parto e a problemas emergenciais do final da gestação.

Um fato lamentável identificado nesta investigação foi o significativo número de mulheres que morreu por complicações do aborto, sendo todos induzidos por razões das pacientes. Isto mostra claramente uma falha nos serviços de saúde na questão do planejamento familiar. A proibição de venda e a dificuldade de obtenção de medicamentos utilizados para promover a interrupção da gestação, provavelmente estão relacionadas ao reaparecimento de casos de abortamento complicado.

As hemorragias constituíram apenas a quarta causa dentre as mortes obstétricas diretas. É reconhecido que estas geralmente constituem a primeira causa em populações menos favorecidas e com maior fecundidade, estando basicamente relacionadas ao atendimento institucional ao parto.

Já as mortes maternas não-obstétricas ou não-relacionadas tiveram um expressivo número de casos atribuiveis à violência, incluindo os acidentes de trânsito e homicídios. Chama a atenção o elevado número de mulheres grávidas que morreu por causas violentas. De fato, o número de mortes por violência foi maior no período que as resultantes de aborto e por hemorragia, o que obriga a um enfoque mais dirigido à violência que atinge dimensões de problema de saúde pública, sobretudo em áreas metropolitanas.

Foi bastante elevado o número de mulheres que tinha patologias prévias à gestação que se agravaram, ou que desenvolveu doenças próprias da gravidez. É de se perguntar, assim, se estas mulheres tivessem recebido uma assistência prénatal adequada, com identificação e controle das patologias associadas, quantas mortes teriam sido evitadas? Todos reconhecem a importância da assistência pré-natal, desde que oferecida em quantidade e qualidade adequadas para toda a população. É importante a captação precoce da gestante aos serviços de saúde, e inclusive da mulher fora da gravidez, para avaliação do seu risco reprodutivo e o seguimento adequado, com possibilidade de referenciá-la para serviços mais capacitados ao atendimento de gestantes de risco. 
Neste estudo aproximadamente 30\% das mulheres que morreram por complicações da gestação, parto e puerpério tinham uma condição patológica associada e, em algumas delas, a possibilidade de engravidar deveria ter sido discutida e provavelmente desaconselhada.

Cabe, por último, discutir até onde os dois casos de coriocarcinoma e os dois suicídios podem ser catalogados como não-relacionados. Todo caso de coriocarcinoma está necessariamente relacionado a uma gestação, apenas podendo se manifestar até muito tempo após seu término. Quanto aos suicídios, apesar de não se ter realizado um estudo mais detalhado de cada um dos casos, é difícil não se considerar a possibilidadede que a decisão de uma mulher terminar com sua vida, estando quase no fim de uma gestação, esteja relacionada justamente com ela. Em assim sendo, estes dois casos têm ainda que nos fazer pensar a que extremos pode chegar uma mulher em nossa sociedade, como conseqüência de uma gravidez.

\section{SUMMARY}

With the purpose of identifying the causes of maternal deaths, this study evaluated all cases of deaths of 10 to 49-years-old women which occurred in Recife, Pernambuco, Brazil, during 1992 and 1993. The data were obtained from 1013 death certificates and were complemented by medical and anesthetia records forms, nursing reports, necropsies and also interviews with physicians who took care of the women, or with their relatives. The main basic causes of maternal deaths identified were arterial hypertension (23.8\%), infections (19.0\%), abortion (11.9\%), hemorrhage (9.5\%), pulmonary embolism (4.8\%) and anaesthetic accident (2.4\%). About $70 \%$ of maternal deaths in Recife in the studied period were due to directo obstetrical causes.

KEY WORDS: Maternal mortality, Hypertension, Puerperal sepsis, Gestational complications.

\section{Referências bibliográficas}

1. Albuquerque RM, Cecatti JG, Hardy E, Faúndes A. Mortalidade materna em Recife 1. Avaliação da subenumeração das estatísticas oficiais. Cad Saúde Públ Rio de Janeiro 1997; 13: 59-65.

2. Boerma J: Levels of maternal mortality in developing countries. Stud Fam Plan 1987; 18: 213-221.
3. Braga LFCO, Nazareno ER, Fanini ML, Carvalho MTW., Soares VMN, Hirata, VM: Relatório do Comitê de Morte Materna do Paraná - 1990. Femina 1992; 20: 186-195.

4. Cecatti JG. Análise da mortalidade materna no município de Campinas, no período de 1985 a 1991. Tese de Doutorado, Campinas, 1992: Universidade Estadual de Campinas.

5. Costa CFF, Maia VO, Lomanchisky G: Mortalidade materna na maternidade Prof. Monteiro de Moraes de 1974 a 1979. Fatores Causais. J Bras Ginecol 1981; 91: 339-341.

6. Faúndes A, Cecatti JG, Silva JLP, Pinotti JA: O problema da mortalidade materna. Femina 1987; 15: 25-31.

7. Ghaham W, Brass W, Snow R: Estimating maternal mortality: The sisterhood method. Stud Fam Plan 1989; 20: 125-135.

8. Henry AO, Shhedy MT, Beischer NA: When is a maternal death a maternal death? A review of maternal death at the Mercy Maternity Hospital, Melbourne. Med J Austr 1989; 151: 628-631.

9. Kaunitz AM, Hughes JM, Grimes DA, Smith JC, Rochat RW, Kafrissen ME: Causes of maternal mortality in the United States. Obstet Gynecol 1985; 65: 605-612.

10. Laurenti R: Marcos referencias para estudos e investigações em mortalidade materna. Rev Saúde Públ 1988; 22: 507-512.

11. Magalhães Netto JM: Mortalidade Materna. Femina 1985; 13: 490-498.

12. Ministério da Saúde: Manual dos Comitês de Mortalidade Materna. Secretaria de Assistência à Saúde, Departamento de Assistência e Promoção à Saúde, Coordenação MaternoInfantil. Brasília, 1994.

13. Organizacion Mundial De La Salud: Guia para el estudio de la mortalidad materna en los paises en desarrollo. Tasas y causas. Ginebra, 1987.

14. Organização Mundial da Saúde: Classificação Estatística Internacional de Doenças e Problemas Relacionados à Saúde. $10^{\mathrm{a}}$ Revisão, Volume 1, Centro Colaborador da OMS para a Classificação de Doenças em Português.São Paulo, 1995.

15. Royston E, Armstrong S: Preventing maternal deaths. World Health Organization, Geneva, 1989.

16. Souza ML: Coeficiente de mortalidade materna segundo o tipo de óbito, grupo etário, paridade, local de residência e tipo de parto. Obituário hospitalar, 1975 a 1979. Florianopólis, SC(Brasil). Rev Saúde Públ, 1983; 17: 279-289. 